\title{
Les gènes SOX : retour vers la détermination du sexe
}

Lors du clonage du gène $S R Y$, ce dernier se révéla appartenir à une nouvelle famille de gènes exprimés au cours du développement embryonnaire, les gènes $S O X[1,2]$. Les gènes SOX dispersés dans le génome et conservés au cours de l'évolution codent pour des facteurs de transcription dont les domaines de liaison à l'ADN présentent une similitude de plus de $60 \%$ avec celui de SRY.

En 1991, pour identifier le produit du gène $S R Y$ au facteur TDF (pour testis determining factor), les génomes de patientes porteuses d'un syndrome de réversion sexuelle furent analysés. Ainsi, chez ces femmes à caryotype $46, \mathrm{XY}$ et porteuses d'une dysgénésie gonadique pure, furent décelées des mutations de novo localisées au cœur même du motif de liaison à l'ADN de la protéine SRY [3].

Si cet argument constitua la preuve génétique de l'égalité SRY = TDF, en fait moins de $20 \%$ des femmes $46, \mathrm{XY}$ présentaient de telles mutations. Qu'en était-il des quelque $80 \%$ restantes? Différentes hypothèses pouvaient être émises sur l'origine moléculaire de la réversion : (1) défaut dans la régulation de l'expression du gène $S R Y$; (2) défaut dans un éventuel partenaire de la protéine SRY ; (3) ou encore altération d'un gène dont le produit intervient dans la bô̂te noire que constituent les étapes situées en aval de l'activation du gène $S R Y$ et qui conduisent à la différenciation des cellules de Sertoli et donc à la production d'hormone anti-Müllérienne.

Pour cloner les gènes de cette boîte noire, sous contrôle ou non de la protéine SRY, l'une des voies privilégiées est l'étude moléculaire des femmes 46,XY chez lesquelles l'inversion du sexe s'accompagne d'une autre malformation.

Ainsi, l'association chez une femme d'un caryotype XY et d'une tumeur de Wilms, qui constitue l'une des formes du syndrome de Denys-Drash, cette cascade d'événements liés au développement sexuel [4]. De même, la dysplasie campomélique caractérisée, entre autres, par de sévères malformations squelettiques, est parfois associée à une inversion sexuelle produisant des femmes XY. $\mathrm{Si}$ la localisation en $17 \mathrm{q}$ du ou des gènes responsables de ce syndrome ne faisait plus aucun doute depuis quelques années, ce n'est que tout récemment que des articles publiés dans les revues Nature (Foster et al., Grande-Bretagne et France [5]) et Cell (Wagner et al., Allemagne, Espagne, Danemark et Norvège [6]) ont décrit l'unique gène impliqué à la fois dans le développement testiculaire et dans la formation des os. Utilisant une technique de clonage positionnel désormais classique, l'analyse de patientes 46XY avec des translocations intéressant le bras long du chromosome 17 (région q23-25) a permis de mettre en cause le gène SOX9 (dont la séquence était partiellement connue) de par sa position distale par rapport au point de cassure du chromosome 17. La description chez plusieurs sujets campoméliques de mutations de novo, sur un seul allèle, confirme l'implication de $S O X 9$, dans ce syndrome autosomique dominant. $\mathrm{Si}$, au contraire de l'intervalle $D S S$ récemment décrit $\left(\mathrm{m} / \mathrm{s} n^{\circ} 11\right.$, vol. $10, p$. 1171), SOX9 intervient dans le développement testiculaire et non ovarien, comme dans le cas de DSS, sa fonction dans la détermination $\mathrm{du}$ sexe est fonction de la dose, exigeant l'expression des deux allèles. SOX9 apparaît comme le premier gène SOX multi-exonique décrit à ce jour et, comme $S R Y$, code pour un facteur de transcription qui, outre un domaine de liaison à l'ADN, possède également un domaine d'activation potentiel, ce qui n'est pas le cas de la protéine SRY humaine.

Le rôle exact de ce nouveau gène dans la cascade de détermination du sexe reste hypothétique. Son produit agit-il directement sous le contrôle de SRY, de concert avec lui, ou encore plus en aval? Quel est son profil d'expression au cours de l'embryogenèse ? Comment relier formation des os et détermination du sexe? Les études en cours chez la souris dans l'équipe de P. Koopman devraient permettre de répondre rapidement à ces questions. L'une des hypothèses séduisantes émises par l'équipe du Pr Goodfellow serait que SOX9 s'exprime, non pas dans les cellules préSertoli, mais dans des cellules mésenchymateuses qui peuvent avoir des devenirs multiples. Aussi, si elles peuvent donner naissance à des cellules constitutives des os et des cartilages, elles peuvent également, après migration à partir du mésonéphros, envahir les cordons génitaux et participer ainsi à la formation des testicules. Après SRY, WT 1, DSS, SF-1 et aujourd'hui $S O X 9$, les différents autres gènes jouant un rôle dans la détermination du sexe masculin devraient être rapidement mis à jour. Gageons que d'autres loci (en $9 p$ ou en $10 q$ par exemple) impliqués dans des délétions chez d'autres femmes XY doivent être aujourd'hui l'objet de nombreuses recherches.

P.B.

1. Gubbay J, Collignon J, Koopman $\mathrm{P}$, Capel B, Economou A, Múnsterberg A, Vivian N, Goodfellow P, Lovell-Badge R. A gene mapping to the sexdetermining region of the mouse Y chromosome is a member of a novel family of embryonically ex-

pressed genes. Nature $1990 ; 346: 245-50$.
2 . Berta $\mathrm{P}$ Gozé C, Poulat F. Mais que sont les 2. Berta P, Gozé C, Poulat F. Mais que sont
gènes SOX? médecine/sciences $1993 ; 9$ ? 1247-8. gènes SOX? médecine/sciences $1993 ; 9$; 1247-8. Griffiths BL, Goodfellow PN, Fellous M. Genetic evidence equating SRY and the testis-determining factor. Nature $1990 ; 348$ : 448-50.

4. Pelletier J, Bruening W, Kashtan CE, Mauer SM, Manivel JC, Striegel JE, Houghton DC, Junien C, Habib R, Fouser L, Fine RN, Silverman BL, Haber DA, Housman D. Germline mutations in the Wilms' tumor suppressor gene are associated with abnormal urogenital development in Denys-Drash abnormal urogenital development

5. Foster. WW Dominguez-Steglich A Guioli S, K ros C Weller PA Stezueg Kwok C, Weller PA, Stevanovic M, Weissenbach J, Mansour S, Young ID, Goodfellow PN, Brook JD, Schafer AJ. Campomelic dysplasia and autosomal sex reversal caused by mutations in an $S R Y$-related gene. Nature $1994 ; 372 ; 525-30$

6. Wagner T, Wirth J, Meyer J, Zabel B, Held M, Zimmer J, Pasantes J, Bricarelli FD, Keutel J, Hustert E, Wolf U, Tommerup N, Schempp W, Scherer G. Autosomal sex reversal and campomelic dysplasia are caused by mutations in and around the $S R Y$ related gene SOX9. Cell 1994; 79 : 1111-20. 